\title{
The changing etiology of liver cancer
}

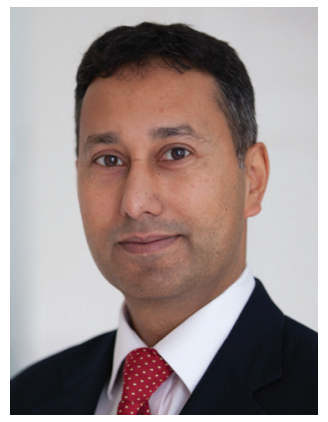

Shahid A Khan* speaks to Georgia Patey, Commissioning Editor: Shahid Khan qualified in Medicine at Guys Hospital Medical School (London, UK) in 1994. His studies in liver cancer led to a PhD from the University of London in 2003 and he was appointed Consultant in Gastroenterology and Hepatology at St Mary's and Hammersmith Hospitals, Imperial College London Healthcare NHS Trust; and a Clinical Senior Lecturer at Imperial College London in 2007. He is currently an Adjunct Reader in the University in addition to being a full-time NHS consultant, is Deputy Admissions Tutor for the Medical School and Director of Clinical Studies at St Mary's Hospital Campus, Imperial College London. He has lectured on liver disease nationally and internationally at hepatology and gastroenterology conferences, including state-of-the art and invited presentations. $\mathrm{He}$ is the lead author on the UK National Guidelines for biliary cancer and was on the International Liver Cancer Association panel for the International Guidelines. He has published extensively in the medical literature, including original research articles, reviews and book chapters and has supervised and examined several postgraduate research degrees in this area. He is a Fellow of the Royal College of Physicians of the UK, and a member of the British Medical Association, the British Association for the Study of the Liver, the British Society of Gastroenterology, the European Association for the Study of the Liver, the American Association for the Study of the Liver Diseases and Chair of the Special Interest Group for non-HCC liver cancers for the International Liver Cancer Association.

Q You are the Academic Lead at Imperial College for Liver Cancer \& a member of the AASLD, BASL, EASL \& ILCA. Can you tell our readers a little about how you became so involved in this field?

I have always been fascinated with cancer as a disease, ever since I was a medical student at Guy's Hospital, London in the early 1990s. So, once I became a Gastroenterology/Hepatology Trainee at Imperial College London, I jumped at the opportunity to do a PhD on liver cancer (1999-2002), under the supervision of Professors Howard Thomas and Simon Taylor-Robinson. My PhD was on the epidemiology, carcinogenic mechanisms and novel biomarkers for cholangiocarcinoma. There was relatively little work in this area at western centers and this led me to being involved in writing national (and later international) guidelines on the diagnosis and management of this type of liver cancer. I have maintained an active and ongoing interest in clinical and research aspects of liver cancer ever since.

*Liver Unit, St Mary's Hospital, Imperial College London/Imperial College Healthcare NHS Trust, London W2 1NY, UK; shahid.khan@imperial.ac.uk

\section{KEYWORDS \\ - diagnosis • etiology \\ - hepatocellular carcinoma \\ - treatment}


Q Today, we are focusing on the changing etiology of liver cancer. What major shifts in etiology of HCC have become apparent over the last 10-20 years, in your experience? What, in your opinion, has led to this?

The major change underway in the etiology of $\mathrm{HCC}$, at least in the western world if not everywhere, is the increasing burden of nonalcoholic fatty liver disease (NAFLD). Fatty liver disease/ metabolic syndrome is probably now the commonest cause of abnormal liver function tests in the west. At least $20-30 \%$ of the population are at risk, and of those people who get NAFLD, around $5 \%$ are likely to get cirrhosis and are at risk of HCC.

A recent study by colleagues from the north of England found HCC-related mortality rose almost twofold in 10 years, from approximately 2 to 4 per 100,000, between 2000 and 2010 [1]. This included a greater than tenfold increase in HCC associated with NAFLD. By 2010, NAFLD accounted for 35\% HCC cases.

Of course viral hepatitis ( $\mathrm{B}$ and $\mathrm{C}$ ) are major causes of HCC worldwide and it will be very interesting to see how the newer highly effective antiHCV drugs will impact HCC incidence over the next few decades, and similarly the highly effective anti-HBV drugs which came out less than a decade ago. Alas, the costs of these drugs means they will be not be readily available to many affected people worldwide in the near future.

\section{Q How has the diagnosis of HCC changed as a result of these changes?}

I do not think the diagnostic pathway in HCC has changed much in the last few years. We are still using imaging, primarily contrast CT MRI and ultrasound, as the major diagnostic tools, although the quality of equipment and technology is increasing all the time. I think there is probably a greater awareness of chronic liver disease being the major risk factor for HCC. There is a also an increasing feeling among some liver specialists that perhaps we should be biopsying HCC more often, at least in the context of advanced HCC in clinical trials, as much histological and potential molecular carcinogenic pathway knowledge has been lost with the reliance on imaging-based diagnosis over the last decade.

\section{Q Similarly, how have the changes impacted} on the treatment of HCC?

The development of novel treatments for HCC has been disappointing over the past half decade and more. The advent of sorafenib was exciting at the time, even though its beneficial effect on survival was modest. However, multiple similar drugs which have been developed and trialed, at great expense, over recent years have shown no greater efficacy. We now know the molecular carcinogenic pathway in HCC is far more complex than previously thought. The failure of the STORM trial to demonstrate that adjuvant Sorafenib reduces recurrence of $\mathrm{HCC}$ after curative ablation or resection was also very disappointing. Given that most HCC patients present with advanced disease, we are desperately in need of new and effective treatments for these patients.

\section{Q Are there any promising new treatments for HCC in the pipeline?}

Novel chemotherapies targeting newly uncovered molecular carcinogenic pathways, the application of newer radiotherapeutic tools, therapeutic vaccines, nanoparticle delivery of antitumor agents and small activating RNA treatments are potentially exciting developments, but the data are currently too small to get overly excited. We must not forget the basics, however, cirrhosis is often preventable if liver disease is diagnosed and managed early on, and the importance of surveillance to detect and treat HCC when they are still potentially curable.

\section{Q What are the major hurdles that we currently face in the field of liver cancer \& how do you think practice/research needs to develop in order to overcome these hurdles over the next 10 years?}

Liver disease generally is on the rise at least in part to the increase in NAFLD. Also, only a fraction of people worldwide with chronic $\mathrm{HBV}$ and $\mathrm{HCV}$ are diagnosed so there is a real concern that HCC incidence could rise over the next couple of decades, despite the real advances in antiviral therapy seen over the last few years. Also, surveillance for HCC in those known to have serious chronic liver disease is haphazard. So there are some basic issues which need addressing at national/international level, involving close liaison between the medical profession, those involved in public health and education and politicians. In terms of research, we need to collaborate more between centers around the world to share resources and biosamples. Working more closely together will help develop more robust answers to relevant research questions, such as: the major pathways involved in hepatocellular carcinogenesis; how these vary 
between different etiologies and ethnicities; and to perform large enough trials of novel therapies to confidently answer questions about their usefulness.

\section{Q Is the rise in $\mathrm{HCC}$ preventable?}

In my opinion, yes the rise in HCC is preventable and even reversible. Worldwide, most cases of chronic liver disease, the major risk factor for HCC, are theoretically preventable. Universal hepatitis $B$ vaccination, early control of chronic active $\mathrm{HBV}$ with currently available drugs, eradication of most cases of HCV, lifestyle changes and public education to deal with the obesity crisis worldwide, addressing alcohol excess, better surveillance, etc. can, if applied internationally and broadly, could hugely reduce the burden of HCC globally. Of course this is easier said than done! Major political and cultural barriers will need to be overcome before this could become a reality. Nonetheless, I remain an optimist!

Financial \& competing interests disclosure

The author has no relevant affiliations or financial involvement with any organization or entity with a financial interest in or financial conflict with the subject matter or materials discussed in the manuscript. This includes employment, consultancies, honoraria, stock ownership or options, expert testimony, grants or patents received or pending, or royalties.

No writing assistance was utilized in the production of this manuscript.

\section{Reference}

1 Dyson J, Jaques B, Chattopadyhay D et al. Hepatocellular cancer: the impact of obesity, Type 2 diabetes and a multidisciplinary team. J. Hepatol. 60(1), 110-117 (2014). 